Technological University Dublin

DÜBLIN

ARROW@TU Dublin

Articles

School of Mathematics

2014

\title{
On commutator socle-regular Abelian p-groups
}

Brendan Goldsmith

Technological University Dublin, brendan.goldsmith@tudublin.ie

Peter Danchev

Plovdiv University

Follow this and additional works at: https://arrow.tudublin.ie/scschmatart

Part of the Mathematics Commons

\section{Recommended Citation}

Goldsmith, B. \& Danchev, P. (2014) On commutator socle-regular Abelian p-groups, Journal of Group Theory, Volume 17, Issue 5, Pages 781-803, ISSN (Online) 1435-4446, ISSN (Print) 1433-5883, DOI:

10.1515/jgt-2014-0003.

This Article is brought to you for free and open access by the School of Mathematics at ARROW@TU Dublin. It has been accepted for inclusion in Articles by an authorized administrator of ARROW@TU Dublin. For more information, please contact arrow.admin@tudublin.ie, aisling.coyne@tudublin.ie,gerard.connolly@tudublin.ie.

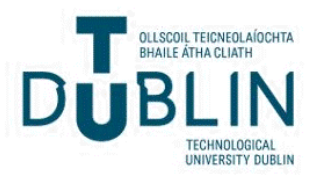




\title{
On commutator socle-regular Abelian p-groups
}

\author{
PETER DANCHEV \\ Department of Mathematics, Plovdiv University \\ "P. Hilendarski", Plovdiv 4000, Bulgaria \\ e-mail: pvdanchev@yahoo.com \\ and \\ BRENDAN GOLDSMITH \\ School of Mathematical Sciences, Dublin Institute of Technology, \\ Aungier Street, Dublin 8, Ireland \\ e-mail: brendan.goldsmith@dit.ie
}

January 5, 2014

\begin{abstract}
We define here the notion of a commutator socle-regular Abelian $p$ group. After establishing some crucial properties of commutator socleregularity, we investigate its relationship with socle-regularity, strong socleregularity and projection socle-regularity.
\end{abstract}

\section{Introduction}

Throughout our discussion we shall focus on additively written Abelian $p$ groups, where $p$ is a prime fixed for the rest of the present paper, although many of the topics we investigate can be considered in a much wider context. The notion of a fully invariant subgroup of a group is, of course, a classical notion in algebra, as is the weaker notion of a characteristic subgroup. Kaplansky devoted a section of his famous "Little Red Book" [16] to the study of such subgroups and, arising from this, he introduced the much-studied classes of transitive and fully transitive groups - see, for example, $[3,4,10]$. Recall that a group $G$ is said to be transitive (respectively, fully transitive) if given $x, y \in G$ with Ulm sequences $\mathrm{U}_{G}(x)=\mathrm{U}_{G}(y)$ (respectively, $\mathrm{U}_{G}(x) \leq \mathrm{U}_{G}(y)$ ), there exists an automorphism (respectively, an endomorphism) $\phi$ such that $\phi(x)=y$. But there are several other weaker notions which have been of interest: recall that a subgroup $H$ of a group $G$ is said to be projection invariant in $G$ if $\pi(H) \leq H$ for all idempotent endomorphisms $\pi$ of $G$ - see, for instance, $[13,18,8,9]$ - while a subgroup $H$ of $G$ is said to be commutator invariant in $G$ if $[\phi, \psi](H) \leq H$ for all $\phi, \psi \in \mathrm{E}(G)$, where, as usual, $[\phi, \psi]$ denotes the additive commutator $\phi \psi-\psi \phi$. These two notions are independent of each other; in fact, there is a commutator

\footnotetext{
${ }^{0} 2010$ AMS Subject Classification: Primary: 20K10, 20K12. Key words and phrases: Abelian $p$-groups, commutator invariant subgroups, projection invariant subgroups, characteristic subgroups, fully invariant subgroups, socle-regular groups, strongly socle-regular groups, projectively socle-regular groups, commutator socle-regular groups.
} 
invariant subgroup that is not projection invariant, and a projection invariant subgroup which is not commutator invariant. For the first case, consider the group $A=\langle a\rangle \oplus\langle b\rangle$ such that $\mathrm{o}(a)=p$ and $\mathrm{o}(b)=p^{3}$ with a proper subgroup $H=\langle a+p b\rangle$. It was established in [2] that $H$ is commutator invariant in $A$ but not a fully invariant subgroup. With the aid of [18] we also deduce that $H$ is not projection invariant in $A$ because in finite groups full invariance and projection invariance coincide. For the second case, the group $G$ of Example 3.2 below will suffice; see the note immediately following the proof of Example 3.3 as well.

In $[5,6]$ the authors generalized the classes of transitive and fully transitive groups by focusing on the possible socles of characteristic and fully invariant subgroups (see [7] too). In [8] full invariance was replaced by projection invariance and the current work continues this theme by replacing full invariance with commutator invariance. Our interest in this was sparked by the timely appearance of Chekhlov's interesting paper [2].

In Section 2 we show that in relation to commutator socle-regularity, one can restrict attention to reduced groups: if $A=D \oplus R$, where $D$ is divisible and $R$ is reduced, then $A$ is commutator socle-regular if, and only if, $R$ is commutator socle-regular- Theorem 2.4. Using realization results of Corner, we establish a useful method of constructing groups whose commutator socle-regularity is precisely determined by that of its first Ulm subgroup. We then exploit this result to show, inter alia, that for groups $G$ with $G / p^{\alpha} G$ totally projective and $\alpha<\omega^{2}$, commutator socle-regularity of $G$ is determined by that of $p^{\alpha} G$ Theorem 2.10; on the other hand we construct groups $G, K$ with $p^{\omega} G=p^{\omega} K$ but $K$ is commutator socle-regular while $G$ is not - Example 2.11.

In section 3 we relate the various notions of socle-regularity that have previously been investigated $([5,6,8])$ with commutator socle-regularity. Our principal results show that the notions are equivalent when the group involved is the direct sum of at least two copies of a fixed group - Theorem 3.5 - but we provide examples showing that the notions are, in fact, different in general. It follows easily from this that summands of commutator socle-regular groups need not be commutator socle-regular- Corollary 3.7. However, we also show that the addition of a separable summand to a group does not influence commutator socle-regularity - Theorem 3.8.

Our interest here will focus on the Abelian p-groups involved but we should point out that a ring-theoretic perspective is also possible: Kaplansky [17] raised the notion of rings in which every element is a sum of additive commutators the so-called commutator rings. These too have been the subject of a great deal of interest; see, e.g., the recent significant work of Mesyan [19].

We re-iterate that all groups throughout the current paper are additively written Abelian $p$-groups, where $p$ is an arbitrary but fixed prime. Our notation and terminology not explicitly stated herein are standard and follow mainly those in [11]. As usual, $\mathrm{E}(G)$ denotes the endomorphism ring of a group $G$. We close this introduction by recalling an important result of A.L.S. Corner [3, Theorem 6.1] which we shall use repeatedly in the sequel: If $H$ is a countable bounded p-group and $\Phi$ is a countable subring of $E(H)$, then $H$ may be imbedded as the subgroup $p^{\omega} G$ of a p-group $G$ such that $E(G)$ acts on $H$ as $\Phi$ and with the property that each $\phi \in \Phi$ extends to an endomorphism $\phi^{*}$ of $G$. The mapping $\phi \mapsto \phi^{*}$ may even be taken as a semigroup homomorphism between the respective multiplicative semigroups of the rings; we shall need this semigroup 
property only in Example 3.3. We shall also exploit the groups constructed by Corner using this imbedding result: there is a fully transitive non-transitive $p$-group with first Ulm subgroup elementary of countably infinite rank and a transitive 2-group which is not fully transitive having a finite first Ulm subgroup which is the direct sum of cycles of order 2 and 8 - see Sections $3 \& 4$ in [4] and [12] for further details.

The construction of examples in this area invariably leads one to considerable amounts of reasonably straightforward but somewhat laborious calculations. These calculations have been recorded separately in an Appendix in order not to interfere with the presentation of results.

\section{The class of commutator socle-regular groups}

In this section we investigate some of the fundamental properties of commutator socle-regular groups; we begin with the appropriate definitions.

Definition 1. A subgroup $C$ of a group $G$ is said to be commutator invariant if $f(C) \leq C$ for every $f \in \mathrm{E}(G)$ which is of the form $f=[\phi, \psi]=\phi \psi-\psi \phi$, where $\phi, \psi \in \mathrm{E}(G)$.

Clearly each fully invariant subgroup is commutator invariant, whereas the converse fails (see, e.g., [2]). Nevertheless, in some concrete situations, commutator invariant subgroups are fully invariant. Specifically, the following result from [2] holds:

Proposition 2.1 (Chekhlov) Suppose $A$ is a group such that $A=\oplus_{i \in I} G$ for some group $G$, where $|I|>1$. Then in $A$ any commutator invariant subgroup is fully invariant.

Proof. We outline an alternative approach to that in [2], utilizing Mesyan's result [19] and some standard matrix representation. Let $H$ be an arbitrary commutator invariant subgroup of $A$. If $|I|$ is infinite, then every element of $\mathrm{E}(A)$ is a sum of commutators - see [19, Theorem 13] - and so if $H$ is commutator invariant, it is then certainly fully invariant.

Suppose then that $A=\bigoplus_{i=1}^{n} G_{i}, n>1$, where each $G_{i} \cong G$, say. Let $E_{i j}(s)$ be the $n \times n$ matrix over the $\operatorname{ring} S=\mathrm{E}(G)$ with $i j^{t h}$-entry equal to $s$ and all other entries zero. Recall that an arbitrary endomorphism of $A$ can be represented as an $n \times n$ matrix $\Delta$ over $S, \Delta=\left(\begin{array}{ccc}d_{11} & \ldots & d_{1 n} \\ \dot{d} & \ldots & \dot{d} \\ d_{n 1} & \ldots & d_{n n}\end{array}\right)$. Now $E_{i j}\left(d_{i j}\right) E_{j j}(1)=E_{i j}\left(d_{i j}\right)$ while $E_{j j}(1) E_{i j}\left(d_{i j}\right)=0$ provided $i \neq j$. So, for $i \neq j, E_{i j}\left(d_{i j}\right)$ is a commutator. Hence $\Delta=\operatorname{diag}\left\{d_{11}, \ldots, d_{n n}\right\}+\Delta^{\prime}$, where $\Delta^{\prime}$ is a sum of commutators. Thus, to establish that $H$ is fully invariant, it suffices to show that $H$ is invariant under the diagonal matrix $\operatorname{diag}\left\{d_{11}, \ldots, d_{n n}\right\}$; in fact, it follows easily that it will suffice to show that $H$ is invariant under the diagonal matrix $\operatorname{diag}\{d, 0, \ldots, 0\}$, where $d=d_{11}$.

Now $E_{n 1}(d)$ is a commutator, so if $\left(g_{1}, \ldots, g_{n}\right)^{t} \in H$ - we are writing elements of $G$ as column vectors and using ()$^{t}$ to denote transposes - then it follows that the matrix product $E_{n 1}(d) \cdot\left(g_{1}, \ldots, g_{n}\right)^{t}=\left(0, \ldots, 0, d g_{1}\right)^{t}$ is also an element of $H$. However, the matrix obtained by interchanging the first and last columns of the identity matrix and 0 elsewhere is also a commutator: 
$E_{1 n}(1)+E_{n 1}(1)=\left[\left(E_{1 n}(1)+E_{n 1}(-1)\right), E_{n n}(1)\right]$. It follows immediately that $\operatorname{diag}\{d, 0, \ldots, 0\} \cdot\left(g_{1}, \ldots, g_{n}\right)^{t}=\left(d g_{1}, 0, \ldots, 0\right)^{t} \in H$ and so $H$ has the required invariance property.

The next result is elementary and we state it without proof for convenience of reference; the content also appears in [2].

Lemma 2.2 (i) If $A$ is a commutator invariant subgroup of the fully invariant subgroup $B$ of a group $C$, then $A$ is commutator invariant in $C$.

(ii) If $A$ is fully invariant in $B$ and $B$ is a a commutator invariant subgroup of $C$, then $A$ is commutator invariant in $C$.

Motivated by similar definitions used previously in $[5,6,8]$, we introduce the following:

Definition 2. A group $G$ is said to be commutator socle-regular if, for each commutator invariant subgroup $C$ of $G$, there exists an ordinal $\alpha$ (depending on $C)$ such that $C[p]=\left(p^{\alpha} G\right)[p]$.

Our first observation is that the property of being commutator socle-regular is inherited by certain subgroups.

Proposition 2.3 If $G$ is a commutator socle-regular group, then so is $p^{\beta} G$ for all ordinals $\beta$.

Proof. Let $C$ be a commutator-invariant subgroup of $p^{\beta} G$. Since the latter is fully invariant in $G$, it follows from Lemma 2.2 that $C$ is commutator invariant in $G$. Consequently, there is an ordinal $\alpha$ such that $C[p]=\left(p^{\alpha} G\right)[p]$. Intersecting both sides of the last equality with $p^{\beta} G$, we obtain that $C[p]=\left(p^{\gamma} G\right)[p]$ where $\gamma=\max (\alpha, \beta)$. But $\gamma=\beta+\delta$ for some $\delta \geq 0$, so that we can write $C[p]=$ $\left(p^{\delta}\left(p^{\beta} G\right)\right)[p]$, as required.

The next result allows us to restrict our attention hereafter to reduced groups.

Theorem 2.4 (i) If $D$ is a divisible group, then $D$ is commutator socle-regular.

(ii) Let $A=D \oplus R$ be a group, where $D$ is a divisible subgroup and $R$ is a reduced subgroup. Then $A$ is commutator socle-regular if, and only if, $R$ is commutator socle-regular.

Proof. (i) If $H$ is a commutator invariant subgroup of $D$, then it follows from Proposition 2.1 that $H$ is fully invariant in $D$. Then $H$ has the form $H=D$ or $H=D\left[p^{n}\right]$ for some non-negative integer $n$ - see, for example, Exercise 68 in $[16]$. Hence, in both situations, we have $H[p]=\left(D\left[p^{n}\right]\right)[p]=D[p]$, as required.

(ii) "Necessity". Suppose that $C$ is an arbitrary commutator invariant subgroup of $R$. We claim that $D \oplus C$ is then a commutator invariant subgroup of A. Assuming we have established this, it follows that $(D \oplus C)[p]=D[p] \oplus C[p]=$ $\left(p^{\alpha} A\right)[p]=\left(p^{\alpha} D\right)[p] \oplus\left(p^{\alpha} R\right)[p]$ for some ordinal $\alpha$. Thus it readily follows that $C[p]=\left(p^{\alpha} R\right)[p]$. Hence it remains only to establish the claim.

Since endomorphisms of $A$ have matrix representations as upper triangular matrices, an easy calculation shows that any commutator homomorphism in $\mathrm{E}(A)$ must have the form $\Delta=\left(\begin{array}{cc}{\left[\alpha, \alpha_{1}\right]} & \delta \\ 0 & {\left[\beta, \beta_{1}\right]}\end{array}\right)$ for endomorphisms $\alpha, \alpha_{1}$ of $D$, 
$\beta, \beta_{1}$ of $R$ and a homomorphism $\delta: R \rightarrow D$. Since $C$ is commutator invariant in $R$, it follows easily that $\Delta(D \oplus C) \leq D \oplus C$, as required.

"Sufficiency". Given that $K$ is an arbitrary commutator invariant subgroup of $A$, Theorem 2 from [2] ensures that $K$ has one of the forms $K=D \oplus C$ or $K=D\left[p^{t}\right] \oplus C$ for some $t \in \mathbb{N} \cup\{0\}$, where in both cases $C$ is a commutator invariant subgroup of $R$. In the first case, $K[p]=D[p] \oplus C[p]=D[p] \oplus\left(p^{\lambda} R\right)[p]=$ $\left(D \oplus p^{\lambda} R\right)[p]=\left(p^{\lambda} D \oplus p^{\lambda} R\right)[p]=\left(p^{\lambda} A\right)[p]$, as desired. For the second case we have $K[p]=\left(D\left[p^{t}\right]\right)[p] \oplus C[p]=D[p] \oplus C[p]=\left(p^{\lambda} A\right)[p]$, as required.

For the remainder of the paper, we shall assume that all groups being discussed, unless explicitly stated to the contrary, are reduced.

We shall made use of the following technical lemma in our next result.

Lemma 2.5 Suppose that $A=\langle a\rangle, B=\langle b\rangle$ are isomorphic cyclic summands of order $p^{n}$ of the group $G$. Then there is a commutator $f$ from $E(G)$ such that $f(a)=b$ or $f(a)=b-s a$, where $s$ is a unit $\bmod p^{n}$.

Proof. Since $A$ is a finite group, it has the exchange property - see, e.g., [11, Theorem 72.1]. Thus if $G=A \oplus N=B \oplus M$, then there exist summands $E_{1}, E_{2}$ of $B, M$ respectively such that $G=A \oplus E_{1} \oplus E_{2}$; let $B=E_{1} \oplus E_{1}^{\prime}, M=E_{2} \oplus E_{2}^{\prime}$ so that $A \cong E_{1}^{\prime} \oplus E_{2}^{\prime}-$ see $\left[11, \S 72\right.$, (a), (b)]. Since $B$ is cyclic, either $E_{1}=\{0\}$ or $E_{1}=B$.

Case (1): If $E_{1}=\{0\}$, then $E_{1}^{\prime}=B$ and so $E_{2}^{\prime}=\{0\}$, implying that $M=E_{2}$. So in this case we have

$$
G=A \oplus M=B \oplus M .
$$

Case (2): If $E_{1}=B$, then

$$
G=A \oplus B \oplus E_{2} .
$$

We now consider the cases separately:

Case (1): $G=A \oplus M=B \oplus M$.

Note that if $a=r b+m$ and $b=s a+m_{1}$ for some $m, m_{1} \in M$, with $r, s$ integers $\bmod p^{n}$, then $a=r s a+\left(r m_{1}+m\right)$, whence we deduce that $r s \equiv 1$ $\bmod p^{n}$. Now define $\phi: G \rightarrow G$ by $\phi(a)=s b, f(m)=0$ for all $m \in M$, and $\psi: G \rightarrow G$ by $\psi(b)=a, \psi(m)=0$ for all $m \in M$. Set $f=\phi \psi-\psi \phi$; a direct calculation shows that $f(a)=b-s a$, as required.

Case (2): $G=A \oplus B \oplus E_{2}$.

Define $\phi: G \rightarrow G$ by $\phi(a)=b, \phi(b)=0$ and $\phi(e)=0$ for all $e \in E_{2}$, and $\psi: G \rightarrow G$ by $\psi(b)=b, \psi(a)=0$ and $\psi(e)=0$ for all $e \in E_{2}$. Set $f=\phi \psi-\psi \phi$; a direct computation shows that $f(a)=b$, as required.

Suppose $H$ is an arbitrary subgroup of the group $G$. Let $\alpha=\min \left\{h_{G}(y)\right.$ : $y \in H[p]\}$ and write $\alpha=\min _{G}(H[p])$; the inclusion $H[p] \leq\left(p^{\alpha} G\right)[p]$ clearly holds. Our next result illustrates some elementary but useful properties of the function $\min _{G}$.

Proposition 2.6 If $C$ is a commutator-invariant subgroup of the group $G$ and $\min _{G}(C[p])=n$, a natural number, then $C[p]=\left(p^{n} G\right)[p]$. 
Proof. Suppose that $C$ is an arbitrary commutator-invariant subgroup of $G$ and $\min _{G}(C[p])=n$, a finite integer. Therefore, there is an element $x \in C[p]$ such that $h_{G}(x)=n$ and so $x=p^{n} y$ where $y$ is the generator of a direct summand of $G$, say $G=\langle y\rangle \oplus G_{1}$; see Corollary 27.2 from [11]. Let $z \in$ $\left(p^{n} G\right)[p] \backslash\left(p^{n+1} G\right)[p]$, so that we write $z=p^{n} w$ for some element $w$ of height zero; thus $G=\langle w\rangle \oplus G_{2}$. Notice that $\langle w\rangle \cong \mathbb{Z}\left(p^{n+1}\right) \cong\langle y\rangle$. By Lemma 2.5, there is a commutator endomorphism $f$ of $G$ such that $f(y)=w$ or $f(y)=w-s y$. Thus we have that $f(x)=z$ or $f(x)=z-s x$ for some $s$. Since $x \in C$ and $C$ is commutator invariant in $G$, either $z \in C$ or $z-s x \in C$; in either case we can conclude that $z \in C$.

If now $z^{\prime}$ is an arbitrary element of $\left(p^{n+1} G\right)[p]$, then $z+z^{\prime} \in\left(p^{n} G\right)[p] \backslash$ $\left(p^{n+1} G\right)[p]$ and so $z+z^{\prime} \in C$, whence $z^{\prime} \in C$. Hence $\left(p^{n} G\right)[p] \leq C$. Since $\min _{G}(C[p])=n$, we certainly have $C[p] \leq\left(p^{n} G\right)[p]$ and so we obtain the desired equality $C[p]=\left(p^{n} G\right)[p]$.

Proposition 2.7 Any large subgroup of a reduced commutator socle-regular group is also commutator socle-regular.

Proof. Let $C$ be a commutator invariant subgroup of a large subgroup $L$ of a commutator socle-regular group $G$. If $\min _{L}(C[p])$ is finite, $n$ say, then it follows from Proposition 2.6 that $C[p]=\left(p^{n} L\right)[p]$. If $\min _{L}(C[p])$ is infinite then so also is $\min _{G}(C[p])$, thus $C[p] \leq\left(p^{\beta} G\right)[p]$ for some infinite ordinal $\beta$. However, utilizing Lemma 2.2, $C$ is commutator invariant in $G$ as well, so $C[p]=\left(p^{\alpha} G\right)[p]$ for some ordinal $\alpha$ and it is immediate that $\alpha \geq \beta$ is infinite. It follows from [1] or $[20, \S 46.1]$ that $p^{\alpha} G=p^{\alpha} L$, whence $C[p]=\left(p^{\alpha} L\right)[p]$. Thus $L$ is commutator socle-regular, as claimed.

An examination of the proof of the proposition above shows that the result holds for any fully invariant subgroup $F$ of a group $G$ having the property that $p^{\omega} F=p^{\omega} G$ (compare also the difference with Example 2.11 below).

Our next proposition is somewhat technical but will enable us to deduce some interesting consequences.

Proposition 2.8 If $G$ is a group with $p^{\omega} G=H$ and for each $\phi \in E(H)$ there is an endomorphism $\phi^{*} \in E(G)$ with $\phi^{*} \uparrow H=\phi$, then $G$ is commutator socleregular if, and only if, $H$ is commutator socle-regular.

Proof. The necessity follows from Proposition 2.3 above.

Conversely, suppose that $H$ is commutator socle-regular and let $C$ be an arbitrary commutator invariant subgroup of $G$. If $\min _{G}(C[p])$ is finite then it follows from Proposition 2.6 that $C[p]=\left(p^{n} G\right)[p]$ for some finite $n$. If $\min _{G}(C[p])$ is infinite, then $C[p] \leq H$. We claim that $C[p]$ is actually a commutator invariant subgroup of $H$. Assuming this for the moment, we conclude, as $H$ is commutator socle-regular, that $C[p]=\left(p^{\alpha} H\right)[p]$ for some ordinal $\alpha$ and hence $C[p]=\left(p^{\alpha}\left(p^{\omega} G\right)\right)[p]=\left(p^{\omega+\alpha} G\right)[p]$, as required.

It remains then to establish the claim. If $f=\phi \psi-\psi \phi$ is any commutator in $\mathrm{E}(H)$, then $f^{*}=\phi^{*} \psi^{*}-\psi^{*} \phi^{*}$ is a commutator in $\mathrm{E}(G)$. But if $x \in H$, then $\left(\phi^{*} \psi^{*}\right)(x)=\phi^{*}(\psi(x))$ since $\psi^{*} \uparrow H-\psi$; note that $y=\psi(x) \in H$ since $\psi \in \mathrm{E}(H)$. Thus $\left(\phi^{*} \psi^{*}\right)(x)=\phi^{*}(y)=\phi(y)=\phi(\psi(x))=(\phi \psi)(x)$ and we have that $\left(\phi^{*} \psi^{*}\right) \uparrow H=\phi \psi$; similarly $\left(\psi^{*} \phi^{*}\right) \uparrow H=\psi \phi$. In particular, if $x \in C[p]$, 
then $f(x)=f^{*}(x) \in C[p]$ since $C$ is a commutator invariant subgroup of $G$ which in turn makes $C[p]$ commutator invariant in $G$. Since $f$ was an arbitrary commutator in $\mathrm{E}(H)$, we conclude that $C[p]$ is a commutator invariant subgroup of $H$, as claimed.

In the proof of our next theorem we shall need an easy extension of a wellknown result on extending automorphisms from the subgroup $p^{n} G, n$ an integer, to automorphisms of the whole group $G$. It is possible to prove this directly using a modification of the argument in [11, Proposition 113.3] but we give here a simple argument which utilizes the result for automorphisms given by Fuchs.

Lemma 2.9 If $n$ is finite and $\phi$ is an arbitrary endomorphism of the subgroup $p^{n} G$ of $G$, then $\phi$ extends to an endomorphism $\phi^{*}$ of $G$.

Proof. Consider the group $H=G \oplus G$ and note that $p^{n} H=p^{n} G \oplus p^{n} G$. Regard endomorphisms of $H$ as $2 \times 2$ matrices over $\mathrm{E}(G)$ and endomorphisms of $p^{n} H$ as $2 \times 2$ matrices over $\mathrm{E}\left(p^{n} G\right)$. Let $\phi \in \mathrm{E}\left(p^{n} G\right)$ be arbitrary. Then $\Delta=\left(\begin{array}{cc}\phi & 1_{p^{n}} G \\ 1_{p^{n}} & 0\end{array}\right)$ is an endomorphism of $p^{n} H$ which is easily seen to actually be an automorphism. By [11, Proposition 113.3], $\Delta$ extends to an automorphism $\Delta^{*}=\left(\begin{array}{cc}\alpha & \gamma \\ \delta & \beta\end{array}\right)$ of $H$, where $\alpha, \beta, \gamma, \delta \in \mathrm{E}(G)$. Thus $\Delta\left(\begin{array}{l}x \\ 0\end{array}\right)=\Delta^{*}\left(\begin{array}{l}x \\ 0\end{array}\right)$ for all $x \in p^{n} H$, i.e., $\left(\begin{array}{c}\phi(x) \\ x\end{array}\right)=\left(\begin{array}{c}\alpha(x) \\ \delta(x)\end{array}\right)$. Set $\phi^{*}=\alpha$, an endomorphism of $G$ and note that $\phi^{*} \uparrow$ $p^{n} G=\alpha \uparrow p^{n} G$, as required.

Our next result indicates, inter alia, that the class of commutator socleregular groups is quite large.

Theorem 2.10 (i) If $G$ is a group such that either $p^{\omega} G=0$ or $p^{\omega} G \cong \mathbb{Z}\left(p^{n}\right)$ for some finite $n$, then $G$ is commutator socle-regular;

(ii) A group $G$ is commutator socle-regular if, and only if, $p^{n} G$ is commutator socle-regular for some $n \in \mathbb{N}$;

(iii) If $G$ is a group such that $G / p^{\alpha} G$ is totally projective for some ordinal $\alpha<\omega^{2}$, then $G$ is commutator socle-regular if, and only if, $p^{\alpha} G$ is commutator socle-regular;

(iv) Totally projective groups of length $<\omega^{2}$ are commutator socle-regular.

Proof. (i) follows immediately from Proposition 2.8 and the observation that in either case the endomorphisms of $p^{\omega} G$ are scalars and hence give rise in a natural way to the desired semigroup homomorphism.

The necessity in (ii) follows directly from Proposition 2.3. The proof of sufficiency is similar to the proof of Proposition 2.8; let $K$ be a commutatorinvariant subgroup of $G$ and if $\min _{G}(K[p])$ is finite, say $m$, then with the aid of Proposition 2.6 we may write $K[p]=\left(p^{m} G\right)[p]$, as required. Otherwise, if $\min _{G}(K[p]) \geq \omega$, then clearly $K[p] \leq p^{\omega} G \leq p^{n} G$. We assert that $K[p]$ is a commutator-invariant subgroup of $p^{n} G$. This follows as in Proposition 2.8 using Lemma 2.9 to deduce that endomorphisms of $p^{n} G$ extend to endomorphisms of $G$. Since $p^{n} G$ is commutator socle-regular, we have that $K[p]=\left(p^{\alpha}\left(p^{n} G\right)\right)[p]$ for some ordinal $\alpha$. Consequently, $K[p]=\left(p^{n+\alpha} G\right)[p]$ and $G$ is commutator socle-regular, as desired.

We will establish (iii) by first considering the case $\alpha=\omega$. In this special case the proof follows from Proposition 2.8 and the observation that as $G / p^{\omega} G$ is 
totally projective, it follows from [15, Theorem 2] that every endomorphism of $p^{\omega} G$ extends to an endomorphism of $G$, thereby giving the extension property required to apply Proposition 2.8 .

Suppose now $\alpha$ has the form $\alpha=\omega \cdot m$ for some $1<m<\omega$. Since $p^{\alpha} G=$ $p^{\omega \cdot m} G=p^{\omega}\left(p^{\omega \cdot(m-1)} G\right)$ is commutator socle-regular and $G / p^{\alpha} G=G / p^{\omega \cdot m} G$ is totally projective, whence so is $p^{\omega \cdot(m-1)}\left(G / p^{\alpha} G\right)=p^{\omega \cdot(m-1)} G / p^{\omega \cdot m} G$, we apply the preceding case $\alpha=\omega$ for $A=p^{\omega \cdot(m-1)} G$ to derive that $p^{\omega \cdot(m-1)} G$ is commutator socle-regular. Moreover, as $G / p^{\alpha} G$ is totally projective so also is $G / p^{\beta} G$ for any $\beta<\alpha$. Thus, after $m-1$ steps, we deduce that $p^{\omega} G$ is commutator socle-regular and $G / p^{\omega} G$ is a direct sum of cyclic groups. Again by what we have shown in the previous paragraph, $G$ will be commutator socleregular, finishing this case.

Finally, consider the case where $\alpha=\omega \cdot m+n$ with $m, n<\omega$. Since $p^{\alpha} G=p^{\omega \cdot m+n} G=p^{n}\left(p^{\omega \cdot m} G\right)$ is commutator socle-regular, we can conclude from (ii) above that the same holds for $p^{\omega \cdot m} G$. As already observed, if $G / p^{\alpha} G$ is totally projective, then so also is $G / p^{\omega \cdot m} G$. We therefore may employ the previous step to conclude that $G$ is commutator socle-regular, indeed.

Part (iv) follows immediately from (iii) by choosing $\alpha$ to be the length of $G$.

Our next example shows that one cannot extend part (i) of the preceding theorem even to the situation where $p^{\omega} G$ is an elementary group of rank 2 .

Example 2.11 There are groups $G, K$ with $p^{\omega} G=\mathbb{Z}(p) \oplus \mathbb{Z}(p)=p^{\omega} K$ where $K$ is commutator socle-regular but $G$ is not.

Proof. Let $H=\langle a\rangle \oplus\langle b\rangle$, where each of $a, b$ is of order $p$. Let $\Phi$ denote the subring of $\mathrm{E}(H)$ consisting (in the usual matrix representation) of the $2 \times 2$ upper triangular matrices $\Delta$ over the field of $p$ elements. A straightforward calculation gives that any commutator in $\Phi$ is strictly upper triangular, i.e., the diagonal entries are also 0. Applying Theorem 6.1 in [3], we find a group $G$ with $p^{\omega} G=H$ such that $\mathrm{E}(G)$ acts on $p^{\omega} G$ as $\Phi$. Consequently, if $\phi$ is any commutator in $\mathrm{E}(G)$, then $\phi$ acts on $p^{\omega} G$ as a strictly upper triangular matrix. In particular, any commutator maps the subgroup $\langle a\rangle$ to 0 and so $\langle a\rangle$ is commutator invariant. But clearly $\langle a\rangle=\langle a\rangle[p]$ cannot have the form $\left(p^{\alpha} G\right)[p]$ for any ordinal $\alpha$ and hence $G$ is not commutator socle-regular.

The construction of $K$ is similar, but this time we take $\Phi$ to be the full endomorphism ring of $H$. An application of Theorem 6.1 in [3] yields a group $K$ with $p^{\omega} K=H$ and a function ()* from $\mathrm{E}(H) \rightarrow \mathrm{E}(K)$ with the properties required to apply Proposition 2.8. Since the finite group $H$ is certainly commutator socle-regular, it follows immediately from Proposition 2.8 that $K$ is also commutator socle-regular.

We remark that it is possible to give a much simpler example than the group $G$ constructed above - for instance, the commutative subring of diagonal matrices would suffice - but, as we shall have need of this particular example later, we have chosen to give this slightly more complicated construction here. 


\section{The various classes of socle-regularity}

In previous works the authors have considered various notions of socle-regularity. These notions have a great degree of similarity since they may be defined in a common way as follows:

A group $G$ is said to be *-socle-regular if every *-subgroup $P$ of $G$ has the property that $P[p]=\left(p^{\alpha} G\right)[p]$ for some ordinal $\alpha$.

When *-subgroup corresponds to fully invariant (characteristic) subgroup, we get the notions that were called socle-regular (strongly socle-regular) groups in $[5,6]$; when *-subgroup corresponds to projection invariant (commutator invariant) subgroup, we get the notion of projectively socle-regular (commutator socle-regular) groups introduced in [8] and the present work respectively.

It is easy to see that the class of socle-regular groups contains each of the other three classes. In this section we investigate the relationships between these different classes; recall that it follows from examples given in $[6,8]$ that the strongly socle-regular and projectively socle-regular classes are properly contained in the class of socle-regular groups. It was also established in [5] that fully transitive groups are socle-regular, while in [6] that transitive groups are strongly socle-regular.

Our first example shows that the class of commutator socle-regular groups is also properly contained in the class of socle-regular groups.

Example 3.1 There is a transitive (and hence strongly socle-regular) group which is neither commutator socle-regular nor projectively socle-regular.

Proof. Let $G$ be the transitive non-fully transitive group constructed as in [4]. Recall that $G$ is a 2-group with $2^{\omega} G=\langle a\rangle \oplus\langle b\rangle=A$, where $o(a)=2, o(b)=8$ and the restriction of $\mathrm{E}(G)$ to $A, \mathrm{E}(G) \uparrow A=\Phi$, where $\Phi$ is the subring generated by the automorphisms of $A$. This group has been thoroughly investigated in [12]; note that the elements of $\Phi$ can be described by two families $\left\{\theta_{i \lambda}\right\}$ and $\left\{\phi_{j \mu}\right\}$ with the parameters $1 \leq i, j \leq 4$ and $\lambda \in\{ \pm 1, \pm 3\}, \mu \in\{0, \pm 1,2\}$. The images of the element $a$ under $\Phi$ are restricted to the possibilities $0, a, 4 b, a+4 b$ and then a straightforward, but somewhat laborious, calculation - see the Appendix for details - shows that every commutator of the form $[\alpha, \beta]$ with $\alpha, \beta \in \Phi$ maps $a \mapsto 0$. We claim that $\langle a\rangle$ is commutator invariant in $G$. For if $[\gamma, \delta]$ is any commutator in $\mathrm{E}(G)$, then $[\gamma, \delta](a)=[\alpha, \beta](a)$ for some $\alpha, \beta \in \Phi$ and so, by the previous observation, we have $[\gamma, \delta](a)=0$. So $\langle a\rangle$ is certainly a commutator invariant subgroup of $G$. However, a direct computation shows that $\langle a\rangle[2]=\langle a\rangle$ is not equal to any of the subgroups $\left(2^{\omega} G\right)[2],\left(2^{\omega+1} G\right)[2],\left(2^{\omega+2} G\right)[2]$ and since $\langle a\rangle$ cannot be of the form $\left(2^{n} G\right)[2]$ for any finite $n$, we conclude that $\langle a\rangle[2] \neq\left(2^{\lambda} G\right)[2]$ for any ordinal $\lambda$ and so $G$ is not commutator socle-regular.

However, $G$ is transitive and hence, by [6, Theorem 4], it is strongly socleregular; moreover, it follows from Proposition 1.13 in [8] that $G$ is not projectively socle-regular.

Our next two examples demonstrate that the classes of commutator socleregular, projectively socle-regular groups and strongly socle-regular groups are distinct.

Example 3.2 There exists a fully transitive commutator socle-regular group that is neither projectively socle-regular nor strongly socle-regular. 
Proof. Suppose that $G$ is the example constructed by Corner in [4] of a non-transitive fully transitive group with $p^{\omega} G \cong H=\bigoplus_{\aleph_{0}} \mathbb{Z}(p)$ and having the property that $\mathrm{E}(G) \uparrow p^{\omega} G=\Phi$ acts as a dense algebra of endomorphisms of $H$. Claim that $G$ is commutator socle-regular.

To see this, let $C$ be an arbitrary commutator invariant subgroup of $G$. If $\min C[p]$ is finite, then with Proposition 2.6 at hand we have that $C[p]=$ $\left(p^{n} G\right)[p]$ for some finite integer $n$; if not, then $C[p] \leq\left(p^{\omega} G\right)[p]$. Now suppose that $0 \neq c \in C[p]$ and let $x$ be an arbitrary element of $\left(p^{\omega} G\right)[p]$ which is linearly independent of $c$. It is straightforward to show that there is a commutator $\phi \in \mathrm{E}(\langle c\rangle \oplus\langle x\rangle)$ with $\phi(c)=x$; say $\phi=f g-g f$ for $f, g \in \mathrm{E}(\langle c\rangle \oplus\langle x\rangle)$. Now, as observed by Corner [4, p. 19], the density property of $\Phi$ means that every endomorphism of a finite subgroup of $p^{\omega} G$ extends to an endomorphism of $G$; in particular $f, g$ extend to mappings $f^{\prime}, g^{\prime}$ of $G$ and so there is a commutator $\phi^{\prime}=f^{\prime} g^{\prime}-g^{\prime} f^{\prime} \in \mathrm{E}(G)$ such that $\phi^{\prime}(c)=\phi(c)=x$. Since $C[p]$ is obviously commutator invariant in $G$, it follows that $x \in C[p]$. Consequently, if the socle of an arbitrary commutator invariant subgroup of $G$ is contained in $p^{\omega} G$, then it must equal $p^{\omega} G$ itself. It now follows immediately that $G$ is commutator socle-regular.

However, $G$ is not projectively socle-regular - see [8, Proposition 1.7] as well as it is not strongly socle-regular - see [6, Theorem 2.3].

Example 3.3 There is a projectively socle-regular group (and hence strongly socle-regular p-group for $p>2$ ) which is not commutator socle-regular.

Proof. We utilize the group $G$ constructed previously in Example 2.11 having $p^{\omega} G=H=\langle a\rangle \oplus\langle b\rangle$, where each of $a, b$ is of order $p$ and where $\mathrm{E}(G)$ acts on $H$ as the subring $\Phi$ of $\mathrm{E}(H)$ consisting (in the usual matrix representation) of the $2 \times 2$ upper triangular matrices $\Delta$ over the field of $p$ elements. We have seen in that example that $G$ is not commutator socle-regular.

We claim, however, that $G$ is projectively socle-regular. Observe firstly that the only idempotent matrices in $\Phi$ are the trivial zero and identity matrices along with the four matrices $\Delta_{1}=\left(\begin{array}{ll}0 & 0 \\ 0 & 1\end{array}\right), \Delta_{2}=\left(\begin{array}{cc}0 & 1 \\ 0 & 1\end{array}\right), \Delta_{3}=\left(\begin{array}{cc}1 & 0 \\ 0 & 0\end{array}\right)$ and $\Delta_{4}=\left(\begin{array}{ll}1 & 1 \\ 0 & 0\end{array}\right)$; this is easily verified by a simple matrix calculation.

Now suppose that $0 \neq P$ is a projection invariant subgroup of $G$. If $\min _{G} P[p]$ is finite, then $P[p]=\left(p^{n} G\right)[p]$ for some finite $n$ by Proposition 1.1 of [8]. If $\min _{G} P[p]$ is infinite, then $P[p]$ is a projection invariant subgroup of $H=p^{\omega} G$. It follows from Corner's construction that if $\pi$ is an idempotent in $\Phi$, then the corresponding extension $\pi^{*} \in \mathrm{E}(G)$ is also an idempotent, since the mapping ()$*$ is actually a semigroup homomorphism from the multiplicative semigroup of $\mathrm{E}(H)$ to that of $\mathrm{E}(G)$, and $\pi^{*} \uparrow H=\pi$. Since $P[p]$ is projection invariant in both $p^{\omega} G$ and $G$, it follows that $\Delta_{i}(P[p]) \leq P[p]$ for $1 \leq i \leq 4$.

Let $(0,0) \neq(u a, v b) \in P[p]$, where $u, v$ are integers. If both $u, v \neq 0$, then applying $\Delta_{1}$ and $\Delta_{3}$ to the element $(u a, v b)$ gives us that both $(u a, 0)$ and $(0, v b)$ belong to $P[p]$ and it follows readily that $P[p]$ must then be all of $H$, i.e., $P[p]=\left(p^{\omega} G\right)[p]$. If $u=0, v \neq 0$, then applying $\Delta_{4}$ to $(0, v b)$ we get that $(v a, 0)$, and hence also $(a, 0)$, belongs to $P[p]$; this again implies that $P[p]=H=H[p]$. If finally $u \neq 0, v=0$, then an identical argument using $\Delta_{2}$ yields the same result. In summary, we deduce that $P[p]=\left(p^{\omega} G\right)[p]$, and $G$ is therefore projectively socle-regular, as required. 
By taking $p \neq 2$, we obtain with Proposition 1.5 of [8] at hand that $G$ is strongly socle-regular, as asserted.

Note that it follows immediately that the group in Example 3.2 has a projection invariant subgroup which is not commutator invariant, while the group in Example 3.3 has a commutator invariant subgroup which is not projection invariant.

Our final example shows that full transitivity is not enough to ensure commutator socle-regularity; our construction is given as a 2-group, but this was merely to simplify calculations and plays no real part.

Example 3.4 There exists a fully transitive (and hence socle-regular) group that is not commutator socle-regular.

Proof. Let $H$ be the finite group $\left\langle a_{1}\right\rangle \oplus\left\langle a_{2}\right\rangle \oplus\left\langle a_{3}\right\rangle$, where $a_{i}$ has order $2^{i}(i=1,2,3)$. Let $e_{i i}$ denote the canonical projection of $H$ onto $a_{i}$; let $\sigma_{i j}(i<j)$ be the forward shift mapping $a_{i} \mapsto 2^{j-i} a_{j}$ and denote by $\tau_{j i}(j>i)$, the backward shift mapping $a_{j} \mapsto a_{i}$. Set $\Phi$ to be the subring of $\mathrm{E}(H)$ generated by $\left\{e_{11},\left(e_{22}+e_{33}\right), \sigma_{12}, \sigma_{13}, \tau_{21}, \sigma_{23}, \tau_{31}, \tau_{32}\right\}$. It is easy to check that the ring generators are linearly independent of additive orders $2,2^{3}, 2,2,2,2^{2}, 2,2^{2}$, so that additively they generate a subgroup of order $2^{12}$. Hennecke [14] has shown that this subring acts fully transitively on $G$ and has order $2^{13}$, so that additively $\Phi$ is not the direct sum of the subgroups generated by the elements listed above. However, the product $\tau_{32} \sigma_{23}=2 e_{22}$ is an element of $\Phi$ and it follows easily that the enlarged set $S=\left\{2 e_{22}, e_{11},\left(e_{22}+e_{33}\right), \sigma_{12}, \sigma_{13}, \tau_{21}, \sigma_{23}, \tau_{31}, \tau_{32}\right\}$ of linearly independent elements generates the ring $\Phi$ additively since the subgroup generated by $S$ has order $2^{13}$. Hence to check the possible actions of commutators from $\Phi$ on $G$, it suffices to consider commutators involving the elements of $S$. Moreover, since a commutator $[\alpha, \beta]=-[\beta, \alpha]$, we can reduce the calculations by half. On the other hand, a straightforward, but rather tedious, direct calculation - see the Appendix - shows that the commutators of the additive generators of $\Phi$ map $a_{2}$ to either $0,2 a_{2}$ or $4 a_{3}$. It follows that the cyclic subgroup $\left\langle 2 a_{2}\right\rangle$ is then mapped to 0 by the commutators of $\Phi$.

Now use Corner's realization result to construct a 2-group $G$ such that $2^{\omega} G=$ $H$ and $\mathrm{E}(G)$ acts on $2^{\omega} G$ as $\Phi$. It follows immediately that $G$ is fully transitive, whence by $[5$, Theorem 0.3$]$ it is socle-regular. Furthermore, the action of $\mathrm{E}(G)$ assures that the subgroup $\left\langle 2 a_{2}\right\rangle$ is commutator invariant in $G$. However, the socle of $\left\langle 2 a_{2}\right\rangle$ is just the subgroup itself since $a_{2}$ has order 4 but $\left(2^{\omega} G\right)[2]=$ $\left\langle a_{1}\right\rangle \oplus\left\langle 2 a_{2}\right\rangle \oplus\left\langle 4 a_{3}\right\rangle,\left(2^{\omega+1} G\right)[2]=\left\langle 2 a_{2}\right\rangle \oplus\left\langle 4 a_{3}\right\rangle,\left(2^{\omega+2} G\right)[2]=\left\langle 4 a_{3}\right\rangle$, so that $\left\langle 2 a_{2}\right\rangle$ is not a socle of the form $\left(2^{\alpha} G\right)[2]$ for any infinite $\alpha$; since it is clearly not of the form $\left(2^{n} G\right)[2]$ for any finite $n$, we conclude that $G$ is not commutator socle-regular, as required.

Nevertheless, in some specific cases, the concepts do coincide. As usual, for each cardinal $\kappa \geq 0$, the $\kappa$-power subgroup $G^{(\kappa)}$ denotes the $\operatorname{direct}$ sum $\oplus_{\kappa} G$ of $\kappa$ copies of $G$.

Theorem 3.5 Let $\kappa>1$. The following conditions are equivalent:

(i) $G$ is socle-regular;

(ii) $G^{(\kappa)}$ is socle-regular; 
(iii) $G^{(\kappa)}$ is strongly socle-regular;

(iv) $G^{(\kappa)}$ is projectively socle-regular;

(v) $G^{(\kappa)}$ is commutator socle-regular.

Proof. In view of Theorem 2.4 of [8], it suffices to obtain only the implication (ii) $\Longleftrightarrow(\mathrm{v})$. The implication $(\mathrm{v}) \Rightarrow$ (ii) is trivial, and the reverse implication follows easily from Proposition 2.1 above.

A direct consequence is the following:

Corollary 3.6 If $G$ is a commutator socle-regular group, then $G^{(\kappa)}$ is commutator socle-regular for any $\kappa \geq 0$.

Proof. As we have seen above, every commutator socle-regular group is socleregular. Thus [5] applies to show that $G^{(\kappa)}$ is socle-regular. We now employ Theorem 3.5 to conclude that this $\kappa$-power group is commutator socle-regular, as desired.

Another consequence is that summands of commutator socle-regular groups need not be again commutator socle-regular.

Corollary 3.7 A summand of a commutator socle-regular group is not necessarily commutator socle-regular.

Proof. Let $G$ be the socle-regular group from Example 3.1 above, which is not commutator socle-regular. However, it follows from Theorem 3.5 that $G \oplus G$ is commutator socle-regular.

Nevertheless, in a certain specific case the following direct summand property holds:

Theorem 3.8 Suppose that $A=G \oplus H$ and $H$ is separable. Then $A$ is commutator socle-regular if, and only if, $G$ is commutator socle-regular.

Proof. Suppose that $G$ is commutator socle-regular and $X$ is a commutator invariant subgroup of $A$. If $\min _{A}(X[p])$ is finite then, by Proposition 2.6, $X[p]=\left(p^{n} A\right)[p]$ for some finite $n$. So, supposing $\min _{A}(X[p])$ is infinite, then $X[p] \leq\left(p^{\omega} A\right)[p]=\left(p^{\omega} G\right)[p]$, as $H$ is separable. However, $X$ is a commutator invariant subgroup of $A$ and so $X[p]$ is a commutator invariant subgroup of $A$ which is actually contained in $G$. Since endomorphisms of $G$ extend trivially to endomorphisms of $A$, it is easy to see that $X[p]$ is actually a commutator invariant subgroup of $G$ and so $X[p]=\left(p^{\lambda} G\right)[p]$ for some ordinal $\lambda$. Thus $\left(p^{\lambda} G\right)[p] \leq\left(p^{\omega} G\right)[p]$ and so $\lambda \geq \omega$. It follows immediately that $X[p]=\left(p^{\lambda} G\right)[p]=\left(p^{\lambda} A\right)[p]$ since $p^{\lambda} H=0$.

Conversely, suppose that $A$ is commutator socle-regular and let $Y$ be an arbitrary commutator invariant subgroup of $G$. As before, if $\min _{G}(Y[p])$ is finite, then Proposition 2.6 assures that $Y[p]=\left(p^{n} G\right)[p]$ for some positive integer $n$. Suppose then that $\min _{G}(Y[p])$ is infinite, so that $Y[p] \leq\left(p^{\omega} G\right)[p]=\left(p^{\omega} A\right)[p]$. We claim that $Y[p]$ is a commutator invariant subgroup of $A$. Assuming for the moment that we have established this claim, it then follows that $Y[p]=\left(p^{\lambda} A\right)[p]$ 
for some ordinal $\lambda$. Hence $Y[p]=\left(p^{\lambda} A\right)[p] \leq\left(p^{\omega} A\right)[p]$, yielding $\lambda \geq \omega$. Since $p^{\lambda} A=p^{\lambda} G$ for $\lambda \geq \omega$, we get the desired result that $Y[p]=\left(p^{\lambda} G\right)[p]$ for some $\lambda$. It remains then only to establish the claim.

Observe firstly that if $\phi=\left(\begin{array}{cc}\alpha & \delta \\ \gamma & \beta\end{array}\right)$ and $\psi=\left(\begin{array}{ll}\alpha_{1} & \delta_{1} \\ \gamma_{1} & \beta_{1}\end{array}\right)$ are arbitrary endomorphisms of $A$ (in the standard matrix representation), then the commutator $[\phi, \psi]$ can be represented as a matrix $\Delta=\left(\begin{array}{cc}{\left[\alpha, \alpha_{1}\right]} & f \\ g & {\left[\beta, \beta_{1}\right]}\end{array}\right)$, where $f: H \rightarrow$ $G, g: G \rightarrow H$ are homomorphisms. Note, however, that as $H$ is separable and $Y[p] \leq\left(p^{\omega} G\right)[p]$, the image under $g$ of each element of $Y[p]$ is necessarily 0 . Identifying $Y[p]$ with $Y[p] \oplus 0$, a straightforward calculation shows that $\Delta(Y[p])=\left[\alpha, \alpha_{1}\right](Y[p])$ and this is clearly contained in $Y[p]$ since $Y$ is, by assumption, a commutator invariant subgroup of $G$.

We finish with a question which we have not been able to resolve at this stage.

- Does there exist a commutator socle-regular group of length $\geq \omega^{2}$; in particular, is the restriction on the ordinal $\alpha$ in Theorem 2.10 (iii) necessary?

Acknowledgement. The authors would like to express their sincere thanks to the referee for helpful suggestions in relation to both technical and presentational issues.

\section{Appendix}

Calculations for Example 3.1 Let $A$ be the finite group defined as $A=\langle a\rangle \oplus\langle b\rangle$, where $a$ has order 2 and $b$ has order 8 . Let $\Phi$ denote the subring of the full endomorphism ring generated by the automorphisms. It is known from [12] that $\Phi$ has order 32 and the elements of $\Phi$ can be labeled as $\left\{\theta_{1 \lambda}, \theta_{2 \lambda}, \theta_{3 \lambda}, \theta_{4 \lambda}\right\}(\lambda= \pm 1, \pm 3)$ and $\left\{\phi_{1 \mu}, \phi_{2 \mu}, \phi_{3, \mu}, \phi_{4 \mu}\right\}(\mu=0, \pm 1,2)$. These are the mappings given by:

- $\theta_{1 \lambda}: a \mapsto a, b \mapsto \lambda b$

- $\theta_{2 \lambda}: a \mapsto a+4 b, b \mapsto \lambda b$

- $\theta_{3 \lambda}: a \mapsto a, b \mapsto a+\lambda b$

- $\theta_{4 \lambda}: a \mapsto a+4 b, b \mapsto a+\lambda b$

- $\phi_{1 \mu}: a \mapsto 4 b, b \mapsto 2 \mu b$

- $\phi_{2 \mu}: a \mapsto 0, b \mapsto a+2 \mu b$

- $\phi_{3 \mu}: a \mapsto 4 b, b \mapsto a+2 \mu b$

- $\phi_{4 \mu}: a \mapsto 0, b \mapsto 2 \mu b$.

In our calculations we shall frequently make use of the following simply verified statement without comment:

if $\lambda, \sigma \in\{ \pm 1, \pm 3\}$ then $\lambda-\sigma$ is even; in particular, if $\lambda \in\{ \pm 1, \pm 3\}$ then $\lambda-1$ is even.

Our objective is to show that for every commutator $[\alpha, \beta]$, where $\alpha, \beta \in \Phi$, we have that $[\alpha, \beta](a)=0$. Clearly we may reduce the amount of calculation by noting that $[\alpha, \beta]=-[\beta, \alpha]$.

(i) Commutators of the form $\left[\theta_{1 \lambda}, \theta_{j \sigma}\right](j \geq 1)$ with $\lambda, \sigma \in\{ \pm 1, \pm 3\}$ :

- $\quad\left[\theta_{1 \lambda}, \theta_{1 \sigma}\right](a)=0$ since $\theta_{1 *}(a)=a$ for all values of $*$;

- $\left[\theta_{1 \lambda}, \theta_{2 \sigma}\right](a)=a+4(\lambda b)-(a+4 b)=4(\lambda-1) b=0$ since $\lambda-1$ is even;

- $\left[\theta_{1 \lambda}, \theta_{3 \sigma}\right](a)=0$ since $\theta_{1 \lambda}(a)=a=\theta_{3 \sigma}(a)$;

- $\left[\theta_{1 \lambda}, \theta_{4 \sigma}\right](a)=a+4 \lambda b-(a+4 b)=0$;

(ii) Commutators of the form $\left[\theta_{2 \lambda}, \theta_{j \sigma}\right](j \geq 2)$ with $\lambda, \sigma \in\{ \pm 1, \pm 3\}$ :

- $\left[\theta_{2 \lambda}, \theta_{2 \sigma}\right](a)=(a+4 b)+4(\lambda b)-\{a+4 b+4(\sigma b)\}=4(\lambda-\sigma) b=0$;

- $\left[\theta_{2 \lambda}, \theta_{3 \sigma}\right](a)=a+4 b-\{a+4(a+\sigma b)\}=4(1-\sigma) b=0$

- $\left[\theta_{2 \lambda}, \theta_{4 \sigma}\right](a)=(a+4 b)+4(\lambda b)-\{a+4 b+4(a+\sigma b)\}=4(\lambda-\sigma) b=0$;

(iii) Commutators of the form $\left[\theta_{3 \lambda}, \theta_{j \sigma}\right](j \geq 3)$ with $\lambda, \sigma \in\{ \pm 1, \pm 3\}$ : 
- $\quad\left[\theta_{3 \lambda}, \theta_{3 \sigma}\right](a)=0$ since $\theta_{3 *}(a)=a$ for all values of $*$;

- $\left[\theta_{3 \lambda}, \theta_{4 \sigma}\right](a)=a+4(a+\lambda b)-(a+4 b)=4(\lambda-1) b=0$;

(iv) Commutators of the form $\left[\theta_{4 \lambda}, \theta_{4 \sigma}\right]$ with $\lambda, \sigma \in\{ \pm 1, \pm 3\}$ :

- $\left[\theta_{4 \lambda}, \theta_{4 \sigma}\right](a)=(a+4 b)+4(a+\lambda b)-\{a+4 b+4(a+\sigma b)\}=4(\lambda-\sigma) b=0$;

Thus we have that all commutators involving pairs of $\theta$ 's map $a \mapsto 0$. Now consider the corresponding situation for the $\phi$ 's.

(v) Commutators of the form $\left[\phi_{1 \mu}, \phi_{j \nu}\right](j \geq 1)$ with $\mu, \nu \in\{0, \pm 1,2\}$ :

- $\left[\phi_{1 \mu}, \phi_{1 \nu}\right](a)=4(2 \mu b)-4(2 \nu b)=0$;

- $\quad\left[\phi_{1 \mu}, \phi_{2 \nu}\right](a)=4(a+2 \mu b)=0$;

- $\left[\phi_{1 \mu}, \phi_{3 \nu}\right](a)=4(2 \mu b)-4(a+2 \nu b)=0$;

- $\left[\phi_{1 \mu}, \phi_{4 \nu}\right](a)=4(2 \mu b)=0$;

(vi) Commutators of the form $\left[\phi_{2 \mu}, \phi_{j \nu}\right](j \geq 2)$ with $\mu, \nu \in\{0, \pm 1,2\}$ :

- $\left[\phi_{2 \mu}, \phi_{2 \nu}\right](a)=0$ since $\phi_{2 *}(a)=0$ for all $* \in\{0, \pm 1,2\}$;

- $\left[\phi_{2 \mu}, \phi_{3 \nu}\right](a)=4(a+2 \mu b)=0$

- $\left[\phi_{2 \mu}, \phi_{4 \nu}\right](a)=0-0=0$;

(vii) Commutators of the form $\left[\phi_{3 \mu}, \phi_{j \nu}\right](j \geq 3)$ with $\mu, \nu \in\{0, \pm 1,2\}$ :

- $\left[\phi_{3 \mu}, \phi_{3 \nu}\right](a)=4(a+2 \mu b)-4(a+2 \nu b)=0$;

- $\left[\phi_{3 \mu}, \phi_{4 \nu}\right](a)=-4(2 \mu b)=0$;

(viii) Commutators of the form $\left[\phi_{4 \mu}, \phi_{4 \nu}\right]$ with $\mu, \nu \in\{0, \pm 1,2\}$ :

- $\left[\phi_{4 \mu}, \phi_{4 \nu}\right](a)=0$ since $\phi_{4 *}(a)=0$ for all $* \in\{0, \pm 1,2\}$;

Thus we have that all commutators involving pairs of $\phi$ 's map $a \mapsto 0$. Now consider the remaining "mixed" situations.

(ix) Commutators of the form $\left[\theta_{1 \lambda}, \phi_{j \nu}\right](j \geq 1)$ with $\lambda \in\{ \pm 1, \pm 3\}, \mu \in\{0, \pm 1,2\}$

- $\left[\theta_{1 \lambda}, \phi_{1 \nu}\right](a)=4(\lambda b)-4 b=4(\lambda-1) b=0$;

- $\left[\theta_{1 \lambda}, \phi_{2 \nu}\right](a)=0-0=0$;

- $\left[\theta_{1 \lambda}, \phi_{3 \nu}\right](a)=4(\lambda b)-4 b=4(\lambda-1) b=0$

- $\left[\theta_{1 \lambda}, \phi_{4 \nu}\right](a)=0-0=0$;

(x) Commutators of the form $\left[\theta_{2 \lambda}, \phi_{j \nu}\right](j \geq 1)$ with $\lambda \in\{ \pm 1, \pm 3\}, \mu \in\{0, \pm 1,2\}$

- $\left[\theta_{2 \lambda}, \phi_{1 \nu}\right](a)=4 \lambda b-\{4 b+4(2 \mu b)\}=4(\lambda-1) b=0$;

- $\left[\theta_{2 \lambda}, \phi_{2 \nu}\right](a)=0-\{0+4(a+2 \mu b)\}=0$;

- $\left[\theta_{2 \lambda}, \phi_{3 \nu}\right](a)=4(\lambda b)-\{4 b+4(a+2 \mu b)\}=4(\lambda-1) b=0$;

- $\left[\theta_{2 \lambda}, \phi_{4 \nu}\right](a)=0-\{0+4(2 \mu b)\}=0$

(xi) Commutators of the form $\left[\theta_{3 \lambda}, \phi_{j \nu}\right](j \geq 1)$ with $\lambda \in\{ \pm 1, \pm 3\}, \mu \in\{0, \pm 1,2\}$

- $\left[\theta_{3 \lambda}, \phi_{1 \nu}\right](a)=4(a+4 b)-4 b=4(\lambda-1) b=0$;

- $\left[\theta_{3 \lambda}, \phi_{2 \nu}\right](a)=0-0$;

- $\left[\theta_{3 \lambda}, \phi_{3 \nu}\right](a)=4(a+\lambda b)-4 b=4(\lambda-1) b=0$;

- $\left[\theta_{3 \lambda}, \phi_{4 \nu}\right](a)=0-0$;

(xii) Commutators of the form $\left[\theta_{4 \lambda}, \phi_{j \nu}\right](j \geq 1)$ with $\lambda \in\{ \pm 1, \pm 3\}, \mu \in\{0, \pm 1,2\}$

- $\left[\theta_{4 \lambda}, \phi_{1 \nu}\right](a)=4(a+\lambda b)-\{4 b+4(2 \mu b)\}=4(\lambda-1) b=0$

- $\left[\theta_{4 \lambda}, \phi_{2 \nu}\right](a)=0-\{0+4(a+2 \mu b)\}=0$;

- $\left[\theta_{4 \lambda}, \phi_{3 \nu}\right](a)=4(a+\lambda b)-\{4 b+4(a+2 \mu b)\}=4(\lambda-1) b=0$;

- $\left[\theta_{4 \lambda}, \phi_{4 \nu}\right](a)=0-\{0+4(2 \mu b)\}=0$

Since the "mixed" commutators also map $a \mapsto 0$, we conclude that every commutator in $\Phi$ maps $a \mapsto 0$ so that the subgroup $\langle a\rangle$ is certainly invariant under the action of commutators from $\Phi$.

Calculations for Example 3.4 Let $G=\left\langle a_{1}\right\rangle \oplus\left\langle a_{2}\right\rangle \oplus\left\langle a_{3}\right\rangle$, where the elements $a_{i}$ have order $2^{i}$. Define the following mappings $G \rightarrow G$ :

- $e_{i i}: a_{i} \mapsto a_{i}, a_{j} \mapsto 0$ if $i \neq j$;

- for $i<j, \sigma_{i j}: a_{i} \mapsto 2^{j-i} a_{j}, a_{k} \mapsto 0$ if $k \neq i$; 
- for $j<i, \tau_{i j}: a_{i} \mapsto a_{j}, a_{k} \mapsto 0$ if $k \neq i$.

Consider the subring $\Phi$ generated (as a ring) by $\left\{e_{11},\left(e_{22}+e_{33}\right), \sigma_{12}, \sigma_{13}, \tau_{21}, \sigma_{23}, \tau_{31}, \tau_{32}\right\}$. It is easy to check that the ring generators are linearly independent of additive orders respectively $2,2^{3}, 2,2,2,2^{2}, 2,2^{2}$, so that additively they generate a subgroup of order $2^{12}$. Hennecke [14] has shown that this subring acts fully transitively on $G$ and has order $2^{13}$, so that additively $\Phi$ is not the direct sum of the subgroups generated by the elements listed above. However, the product $\tau_{32} \sigma_{23}=2 e_{22}$ is an element of $\Phi$ and it follows easily that the enlarged set $S=\left\{2 e_{22}, e_{11},\left(e_{22}+e_{33}\right), \sigma_{12}, \sigma_{13}, \tau_{21}, \sigma_{23}, \tau_{31}, \tau_{32}\right\}$ of linearly independent elements generates the ring $\Phi$ additively since the subgroup generated by $S$ has order $2^{13}$. Hence to check the possible actions of commutators from $\Phi$ on $G$, it suffices to consider commutators involving the elements of $S$. Moreover, since a commutator $[\alpha, \beta]=-[\beta, \alpha]$, we can reduce the calculations by half.

We consider actions of commutators from $S$ on the subgroup $\left\langle a_{2}\right\rangle$.

(i) Commutators involving $\tau_{32}$ :

- $\left[\tau_{32}, \tau_{31}\right]\left(a_{2}\right)=\tau_{32}(0)-\tau_{31}(0)=0$;

- $\left[\tau_{32}, \sigma_{23}\right]\left(a_{2}\right)=\tau_{32}\left(2 a_{3}\right)-\sigma_{23}(0)=2 a_{2}-0=2 a_{2}$;

- $\left[\tau_{32}, \tau_{21}\right]\left(a_{2}\right)=\tau_{32}\left(a_{1}\right)-\tau_{21}(0)=0$

- $\left[\tau_{32}, \sigma_{13}\right]\left(a_{2}\right)=\tau_{32}(0)-\sigma_{13}(0)=0$;

- $\left[\tau_{32}, \sigma_{12}\right]\left(a_{2}\right)=\tau_{32}(0)-\sigma_{12}(0)=0$; $\left[\tau_{32}, e_{33}\right]\left(a_{2}\right)=\tau_{32}(0)-e_{33}(0)=0$

$\left[\tau_{32}, e_{22}\right]\left(a_{2}\right)=\tau_{32}\left(a_{2}\right)-e_{22}(0)=0 ;$

- Hence $\left[\tau_{32},\left(e_{33}+e_{22}\right)\right]\left(a_{2}\right)=0$;

- $\left[\tau_{32}, e_{11}\right]\left(a_{2}\right)=\tau_{32}(0)-e_{11}(0)=0$;

- $\left[\tau_{32}, 2 e_{22}\right]\left(a_{2}\right)=2\left[\tau_{32}, e_{22}\right]\left(a_{2}\right)=0$.

(ii) Commutators involving $\tau_{31}$ :

- $\left[\tau_{31}, \sigma_{23}\right]\left(a_{2}\right)=\tau_{31}\left(2 a_{3}\right)-\sigma_{23}(0)=2 a_{1}=0$;

- $\left[\tau_{31}, \tau_{21}\right]\left(a_{2}\right)=\tau_{31}\left(a_{1}\right)-\tau_{21}(0)=0$

- $\left[\tau_{31}, \sigma_{13}\right]\left(a_{2}\right)=\tau_{31}(0)-\sigma_{13}(0)=0$;

- $\left[\tau_{31}, \sigma_{12}\right]\left(a_{2}\right)=\tau_{31}(0)-\sigma_{12}(0)=0$;

$\left[\tau_{31}, e_{33}\right]\left(a_{2}\right)=\tau_{31}(0)-e_{33}(0)=0$

$\left.\left[\tau_{31}, e_{22}\right]\left(a_{2}\right)=\tau_{31}\left(a_{2}\right)\right)-e_{22}(0)=0 ;$

- Hence $\left[\tau_{31},\left(e_{33}+e_{22}\right)\right]\left(a_{2}\right)=0$;

- $\quad\left[\tau_{31}, e_{11}\right]\left(a_{2}\right)=\tau_{31}(0)-e_{11}(0)=0$;

- $\left.\left.\left[\tau_{31}, 2 e_{22}\right)\right]\left(a_{2}\right)=2\left[\tau_{31}, e_{22}\right)\right]\left(a_{2}\right)=0$.

(iii) Commutators involving $\sigma_{23}$ :

- $\left[\sigma_{23}, \tau_{21}\right]\left(a_{2}\right)=\sigma_{23}\left(a_{1}\right)-\tau_{21}\left(2 a_{3}\right)=0$;

- $\left[\sigma_{23}, \sigma_{13}\right]\left(a_{2}\right)=\sigma_{23}(0)-\sigma_{12}\left(2 a_{3}\right)=0$;

$\left[\sigma_{23}, e_{33}\right]\left(a_{2}\right)=\sigma_{23}(0)-e_{33}\left(2 a_{3}\right)=-2 a_{33} ;$

$\left[\sigma_{23}, e_{22}\right]\left(a_{2}\right)=\sigma_{23}\left(a_{2}\right)-e_{22}\left(2 a_{3}\right)=2 a_{3}$;

- Hence $\left[\sigma_{23},\left(e_{33}+e_{22}\right)\right]=-2 a_{3}+2 a_{3}=0$;

- $\quad\left[\sigma_{23}, e_{11}\right]\left(a_{2}\right)=\sigma_{23}(0)-e_{11}\left(2 a_{3}\right)=0$;

- $\left[\sigma_{23}, 2 e_{22}\right]\left(a_{2}\right)=2\left[\sigma_{23}, \varepsilon_{22}\right]\left(a_{2}\right)=0$;

(iv) Commutators involving $\tau_{21}$ :

- $\left[\tau_{21}, \sigma_{13}\right]\left(a_{2}\right)=\tau_{21}(0)-\sigma_{13}\left(a_{1}\right)=4 a_{3}$;

- $\left[\tau_{21}, \sigma_{12}\right]\left(a_{2}\right)=\tau_{21}(0)-\sigma_{12}\left(a_{1}\right)=-2 a_{2}=2 a_{2}$; $\left[\tau_{21}, e_{33}\right]\left(a_{2}\right)=\tau_{21}(0)-e_{33}\left(a_{1}\right)=0$;

$\left[\tau_{21}, e_{22}\right]\left(a_{2}\right)=\tau_{21}\left(a_{2}\right)-e_{22}\left(a_{1}\right)=-a_{1}=a_{1}$;

- Hence $\left[\tau_{21},\left(e_{33}+e_{22}\right]\left(a_{2}\right)=-a_{1}=a_{1}\right.$;

- $\left[\tau_{21}, e_{11}\right]\left(a_{2}\right)=\tau_{21}(0)-e_{11}\left(a_{1}\right)=-a_{1}=a_{1}$;

- $\left[\tau_{21}, 2 e_{22}\right]\left(a_{2}\right)=2\left[\tau_{21}, e_{22}\right]\left(a_{2}\right)=2 a_{1}=0$.

(v) Commutators involving $\sigma_{13}$ :

- $\left[\sigma_{13}, \sigma_{12}\right]\left(a_{2}\right)=\sigma_{13}(0)-\sigma_{12}(0)=0$;

$$
\left[\sigma_{13}, e_{33}\right]\left(a_{2}\right)=\sigma_{13}(0)-e_{33}(0)=0 ;
$$

$\left[\sigma_{13}, e_{22}\right]\left(a_{2}\right)=\sigma_{13}\left(a_{2}\right)-e_{22}(0)=0$;

- Hence $\left[\sigma_{13},\left(e_{33}+e_{22}\right)\right]\left(a_{2}\right)=0$;

- $\left[\sigma_{13}, e_{11}\right]\left(a_{2}\right)=\sigma_{13}(0)-e_{11}(0)=0$; 
- $\left[\sigma_{13}, 2 e_{22}\right]\left(a_{2}\right)=2\left[\sigma_{13}, e_{22}\right]=0$.

(vi) Commutators involving $\sigma_{12}$ :

$$
\begin{gathered}
{\left[\sigma_{12}, e_{33}\right]\left(a_{2}\right)=\sigma_{12}(0)-e_{33}(0)=0 ;} \\
{\left[\sigma_{12}, e_{22}\right]\left(a_{2}\right)=\sigma_{12}\left(a_{2}\right)-e_{22}(0)=0 ;} \\
\text { - Hence }\left[\sigma_{12},\left(e_{33}+e_{22}\right)\right]\left(a_{2}\right)=0 ; \\
-\quad\left[\sigma_{12}, e_{11}\right]\left(a_{2}\right)=\sigma_{12}(0)-e_{11}(0)=0 ; \\
-\quad\left[\sigma_{12}, 2 e_{22}\right]\left(a_{2}\right)=2\left[\sigma_{12}, e_{22}\right]\left(a_{2}\right)=0 .
\end{gathered}
$$

(vii) Commutators involving $\left(e_{22}+e_{33}\right)$ :

$$
\begin{aligned}
& {\left[e_{33}, e_{11}\right]\left(a_{2}\right)=e_{33}(0)-e_{11}(0)=0 ;} \\
& {\left[e_{22}, e_{11}\right]\left(a_{2}\right)=e_{22}(0)-e_{11}\left(a_{2}\right)=0 ;}
\end{aligned}
$$

- Hence $\left[\left(e_{22}+e_{33}\right), e_{11}\right]\left(a_{2}\right)=0$.

(viii) Commutators involving $e_{11}$ :

- $\quad\left[e_{11}, 2 e_{22}\right]\left(a_{2}\right)=2\left[e_{11}, e_{22}\right]\left(a_{2}\right)=0$.

It follows from the calculations above that the images of $a_{2}$ under the elements of $\Phi$ belong to the set $\left\{0, a_{1}, 2 a_{2}, 4 a_{3}\right\}$. Thus the subgroup $\left\langle 2 a_{2}\right\rangle$ is mapped to 0 by $\Phi$; in particular $\left\langle 2 a_{2}\right\rangle$ is invariant under commutators from $\Phi$, as required.

\section{References}

[1] Kh. Benabdallah, B. Eisenstadt, J. Irwin and M. Poluianov, The structure of large subgroups of primary Abelian groups, Acta Math. Acad. Sci. Hungar. (3-4) 21 (1970), 421-435.

[2] A.R. Chekhlov, Commutator invariant subgroups of Abelian groups, Siber. Math. J. (5) 51 (2010), 926-934.

[3] A.L.S. Corner, On endomorphism rings of primary Abelian groups II, Quart. J. Math. Oxford 27 (1976), 5-13.

[4] A.L.S. Corner, The independence of Kaplansky's notions of transitivity and full transitivity, Quart. J. Math. Oxford (2) 27 (1976), 15-20.

[5] P. Danchev and B. Goldsmith, On the socles of fully invariant subgroups of Abelian p-groups, Arch. Math. (3) 92 (2009), 191-199.

[6] P. Danchev and B. Goldsmith, On the socles of characteristic subgroups of Abelian p-groups, J. Algebra (10) 323 (2010), 3020-3028.

[7] P. Danchev and B. Goldsmith, On socle-regularity and some notions of transitivity for Abelian p-groups, J. Comm. Algebra (3) 3 (2011), 301-319.

[8] P. Danchev and B. Goldsmith, On projection-invariant subgroups of Abelian p-groups, in Groups and Model Theory, Contemporary Mathematics, vol. 576, Amer. Math. Soc., Providence, RI, 2012, 31-40.

[9] P. Danchev and B. Goldsmith, On projectively fully transitive Abelian pgroups, Results Math. 63 (2013), 1109-1130.

[10] S. Files and B. Goldsmith, Transitive and fully transitive groups, Proc. Amer. Math. Soc. 126 (1998), 1605-1610. 
[11] L. Fuchs, Infinite Abelian Groups, Vol. I and Vol. II, Academic Press, New York and London, 1970 and 1973.

[12] B. Goldsmith and L. Strüngmann, Some transitivity results for torsion Abelian groups, Houston J. Math. 23 (2007), 941-957.

[13] J. Hausen, Endomorphism rings generated by idempotents, Tamkang J. Math. 9 (1978), 215-218.

[14] G. Hennecke, Unpublished calculations relating to the thesis Transitive und volltransitive Abelsche p-gruppen, Diplomarbeit im Fachbereich Mathematik an der Universität GH Essen 1996.

[15] P. Hill, On transitive and fully transitive primary groups, Proc. Amer. Math. Soc. 22 (1969), 414-417.

[16] I. Kaplansky, Infinite Abelian Groups, University of Michigan Press, Ann Arbor, 1954 and 1969.

[17] I. Kaplansky, Problems in the theory of rings revisited, Amer. Math. Monthly 77 (1970), 445-454.

[18] C. Megibben, Projection-invariant subgroups of Abelian groups, Tamkang J. Math. 8 (1977), 177-182.

[19] Z. Mesyan, Commutator rings, Bull. Austral. Math. Soc. 74 (2006), 279 288.

[20] L. Salce, Struttura dei p-gruppi abeliani, Pitagora Ed., Bologna, 1980. 